\title{
Association of Intraoperative Cerebral and Somatic Tissue Oxygen Saturation with Postoperative Cognitive Dysfunction After Spine Open Surgery in Patients with Hypertension: An Observational Study
}

Fei Guo

Department of Anesthesiology, Zigong Fourth People's Hospital Affiliated to Southwest Medical University,Zigong 643000,Sichuan,China

\section{Shuaiying Jia}

Department of Anesthesiology,Zigong Fourth People's Hospital Affiliated to Southwest Medicalm University,Zigong 643000,Sichuan,Chian

\section{Qiyan Wang}

Department of Anesthesiology,Zigong Fourth People's Hospital Affiliated to Southwest Medical University,Zigong 643000,Sichuan,China

Qinyu Liu

Translational Medicine Center,the Second Affiliated Hospital,Guangzhou Medical University,Guangzhou510260,Guangdong,China

\section{Mingquan $\mathrm{Hu}$}

Department of Anesthesiology,Zigong Fourth People's Hospital Affiliated to Southwest Medicine University,Zigong 643000,Sichuan,China

\section{Wenzhang Wang}

Department of Anesthesiology,Zigong Fourth People's Hospital Affiliated to Southwest Medical University,Zigong 643000,Sichuan,China

\section{Shijian Liu}

Department of Anesthesiology,Zigong Fourth People's Hospital Affiliated to Southwest Medical University,Zigong 643000,Sichuan,China

\section{Qiang Li}

Department of Anesthesiology,Zigong Fourth People's Hospital Affiliated to Southwest Medical University,Zigong 643000,Sichuan,China

\section{Bin Lu}

Department of Anesthesiology,Zigong Fourth People's Hospital Affiliated to Southwest Medical University,Zigong 643000,Sichuan,China

\section{Yeying Zheng ( $\nabla$ walfb@126.com )}

department of Anesthesiolog,zi gong fourth peoples hospital https://orcid.org/0000-0002-4564-5975 
Research

Keywords: postoperative cognitive dysfunction, spine surgery, cerebral oxygen saturation, near-infrared spectroscopy, muscular oxygen saturation, hypertension

Posted Date: September 20th, 2021

DOl: https://doi.org/10.21203/rs.3.rs-903012/v1

License: (c) (i) This work is licensed under a Creative Commons Attribution 4.0 International License.

Read Full License 


\section{Abstract}

Background: Intraoperative cerebral desaturations have been associated with worse neurological outcomes after supine surgery. However, it is not clear whether intraoperative somatic tissue oxygenation is more associated with postoperative cognitive dysfunction (POCD) than cerebral oxygenation in patients with hypertension after prone spine surgery.

Methods: Patients with hypertension scheduled for spine open surgery were included from 2020 to 2021 in a single-center, prospective, observational study. Baseline both cerebral and somatic tissue oxygen saturation were measured in operating room before surgery. Cerebral and somatic tissue oxygen saturations were monitored continuously throughout surgery. The presence of POCD was assessed using the Mini-Mental Status Examination (MMSE). Association with POCD was evaluated with unadjusted analyses and multivariable logistic regression.

Results: One hundred and one of 112 patients were included, and 28 (27.8\%) developed POCD. None of the investigated SctO2 variables was predictive of POCD. On the contrary, the patients with POCD had a higher decrease in intraoperative absolute Sst02 decrease and relative Sst02 decrease compared with the patients without POCD $(4.9 \% \pm 3.8 \%$ vs. $3.6 \% \pm 2.6 \%, P=0.037 ; 7.4 \% \pm 5.6 \%$ vs. $5.3 \% \pm 3.8 \%, P=0.036$; respectively). Finally, three Sst02 parameters respectively were associated with $\mathrm{POCD}$, including a higher absolute SstO2 decrease (OR, 1.223; 95\%Cl, 1.031-1.451; $\mathrm{P}=0.021)$, a higher absolute SstO2 decrease (OR, $1.138 ; 95 \% \mathrm{Cl}, 1.011-1.281 ; \mathrm{P}=0.032)$ and falling below $90 \%$ of baseline SstO2 (OR, 11.388; $95 \% \mathrm{Cl}, 2.367-$ 54.785; $\mathrm{P}=0.002)$, independent of ASA III, preoperative platelet and postoperative sepsis.

Conclusions: Twenty-eight (27.8\%) of 101patients developed POCD. Somatic tissue oxygenation has a stronger association with POCD than cerebral tissue oxygenation after spine open surgery in patients with hypertension.

Clinical trial registration: ChiCTR1900028392. Registered 20 December 2019.

\section{Background}

Patients with hypertension undergoing spine open surgery are at risk of developing postoperative cognitive dysfunction (POCD).$^{[1]}$ POCD has been associated with a prolonged hospital stay, long-term neurocognitive deterioration, increased mortality, and increased social costs. ${ }^{[2-4]}$ No anesthetic or surgical strategy, at present, has been proven to minimize cognitive impact. ${ }^{[5]}$ Identifying predictors for the development of POCD is therefore vital in the early prevention and treatment of this condition. ${ }^{[4,6]}$ Intraoperative hypoxia and hypoperfusion have frequently been regarded as important factors contributing to the development of POCD. ${ }^{[1,7]}$ Near-infrared spectroscopy (NIRS) can noninvasively measure the regional cerebral oxygen saturation (SctO2) and/or somatic tissue oxygen saturation (Sst02) at the microvascular level, thereby enabling the detection of mismatches between oxygen supply

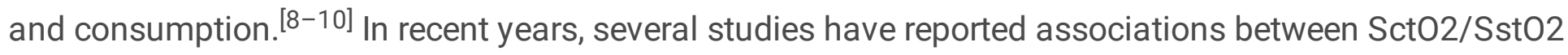


and neurological complications. ${ }^{[11-16]}$ However, of these studies, patients were in a supine position in most studies, patients were in a prone position in only one study and only SctO2 was monitored, ${ }^{[11]}$ so the relationship between Sct02/SstO2 and POCD in prone surgery remains unclear. A study, in prone spine surgery recently, had shown a good association between postoperative complications and Sst02, but almost no association with Sct02. ${ }^{[17]}$ As such, we hypothesized that in a prone position, SctO2 is affected by position but SstO2 is not. The aim of this prospective observational study was to evaluate the association between intraoperative Sct02/SstO2 and POCD after open prone spine surgery in patients with hypertension.

\section{Methods}

This prospective observational study was conducted in a tertiary hospital. The study protocol was approved by the Institutional Committee for Medical Ethics of Zigong Fourth People's Hospital Affiliated to Southwest Medical University, Zigong, Sichuan, China. Written informed consent was obtained from the patient before surgery.

\section{Study population}

Patients undergoing elective posterior lumbar or/and thoracic spine open surgery in a prone position were enrolled between January 2020 and April 2021. Our goal was to recruit 100 patients in this study, as the previous observational studies assessing the association between Sct02/Sst02 and postoperative outcomes had approximately 100 patients in whom monitoring only was implemented. ${ }^{[13,17]}$ The exclusion criteria included: 1) patient refusal; 2) age < 18 years old; 3) American Society of Anesthesiologists (ASA) physical status score > III; 4) combined with other operations; 5) skin condition affecting the monitoring of the oximetry probe; 6 ) preoperative Mini-Mental State Examination (MMSE) score $<18$; 7) literacy problems, language difficulties, hearing or visual impairment.

\section{Anesthesia}

No premedication was given. After patients entered operating room, radial arterial cannulation, arterial blood gas analysis and the monitoring of bispectral index (BIS) monitoring were performed in advance under the breathing room air. General anesthesia was induced using midazolam $(0.05 \mathrm{mg} / \mathrm{kg})$, sufentanil (0.3-0.4 ug/ $\mathrm{kg})$, cisatracurium $(0.2 \mathrm{mg} / \mathrm{kg})$ and etomidate $(0.2 \mathrm{mg} / \mathrm{kg})$. Then propofol $(0-1.5 \mathrm{mg} / \mathrm{kg}) \mathrm{was}$ used according to the BIS before tracheal intubation was performed. At the administration of the same attending anesthesiologist, general anesthesia was maintained with BIS from 40-60\% using sevoflurane in an oxygen-air mixture (oxygen concentration 40\%). Respiratory rate was set to meet the end-tidal CO2 35-40 mmHg. Sufentanil and cisatracurium were added intermittently by the attending anesthesiologist according to the needs of the surgery. Ephedrine was usually used to maintain blood pressure, and when ephedrine did not work well, deoxyepinephrine was used. Tranexamic acid was routinely applied 30 minutes before surgical skin cutting. Cell salvaging was available in all patients. After surgery, analgesia 
through incision infiltration was routinely performed, and oral analgesia drugs and/or self-controlled electronic analgesia pump were administered as needed.

\section{Tissue oxygenation monitoring}

Tissue oxygenation was monitored using a tissue oximeter (EGOS-600A, Eqin tissue oxygen saturation monitor, China) based on NIRS. The oximeter had four cables with each cable connected to an NIRS sensor. Two NIRS sensors were placed on the upper forehead, one finger above the eyebrow, to monitor the left and right frontal lobe Sct02, respectively. The other two NIRS sensors were placed on the waist in the midaxillary line, two fingers below the costal margin, to monitor the SstO2 of the left and right waist, respectively. All four NIRS sensors were placed on the patient when entering the operating room. The patient's baseline SctO2 and SstO2 were acquired before anesthetic induction when the patient breathed air. The monitoring and data recording were started when the anesthetic induction was begun, and were stopped when the operation was done. The oximeter was put at the back of anesthesia machine and blinded to anesthesiologists.

The tissue oximeter generated a new data point every two seconds that was continuously recorded by a computer. The data points of Sct02/ SstO2 were used for tissue oxygenation indices derivation. Eight indices of tissue oxygenation were derived that include the baseline, minimum, mean, median, absolute SctO2 decrease, relative SctO2 decrease, area under the curve (AUC) and number of desaturation $<80 \%$ or $<90 \%$ of baseline. The AUC was the sum of the differences between the special threshold and the data point of every minute that was less than the threshold. For each index, SctO2 and SstO2 were treated independently.

\section{Clinical covariates}

Patient characteristics including age, sex, weight, height, current smoke, alcohol consumption and ASA were collected. Past medical history including diagnosis of hypertension, coronary artery disease, previous stroke, diabetes mellitus, COPD, chronic hepatitis B and chronic kidney disease were recorded. Surgical information that were recorded were fusion or not, number of segments, estimated blood loss and surgical time. The usage of ephedrine and phenylephrine, fluid infusion volume, lactate when suturing, urine output and blood transfusion were recorded. After surgery, the time of tracheal extubation, length of PACU stay, numerical pain score (NPS, in postoperative1d and 3d), and postoperative $24 \mathrm{~h}$ drainage volume were also recorded.

\section{Postoperative outcomes}

The primary outcome measure was POCD. The testing protocol was the quick assessment scales of the mini-mental state examination (MMSE) that used widely as a screening tool for cognitive dysfunction. [18] MMSE involves testing orientation to time, place, registration, attention, calculations, recall, repetition, and complex commands. ${ }^{[18]}$ It is known to be less sensitive when evaluated repeatedly. ${ }^{[19]}$ Therefore, in this study, cognitive function tests were performed only on day 1 before surgery and day 4 after surgery, 
respectively. When administering the MMSE a second time, variation in the order of test elements was used to increase the sensitivity of the test.

The testing of cognitive function was performed by a special clinician with training in cognitive assessment. The testing clinician was blinded to surgery and anesthesia for each patient. In this study, a decrease by 2 points or more from the preoperative score was considered as a significant decline in cognitive function, and defined as a POCD. ${ }^{[11]}$

Primary postoperative complications were identified based on chart review of the electronic medical records at the Zigong fourth People's Hospital Affiliated to Southwest Medical University. Information of length of hospital stay, length of postoperative hospital stay and total medical costs were also recorded.

\section{Statistical analysis}

Statistical analysis was performed using IBM SPSS version 23 (IBM, Chicago, IL). Normal distribution of continuous data was evaluated with the Kolmogorove-Smirnov test. Normally or approximately normally distributed data are presented as mean ( \pm standard deviation), and skewness distributed data are presented as median with first and third quartiles. Categorical variables are expressed as $\mathrm{n}(\%)$.

Continuous data were compared by independent t-test or Mann-Whitney U-test, and categorical data were compared between groups with and without POCD using chi-square test or Fisher's exact test. Repeated measurements between two groups were compared by paired t-test. Correlation between two continuous variables was analyzed using Spearman or Pearson correlation.

Baseline patient characteristics and perioperative characteristics that were significant (at a threshold $\mathrm{P}<$ 0.2 ) in the univariable logistic regression analysis were entered into a forward multivariable logistic regression model. The multicollinearity test was performed on all included variables in advance, and variables with a variance inflation factor $>5$ were excluded. The goodness of fit of the final multivariable logistic regression model was evaluated using the Hosmere-Lemeshow test. The predictive power of the final model was assessed by a receiver operating characteristic (ROC) curve, and the AUC was calculated to evaluate the performance in predicting POCD. Finally, to clarify the relationship between Sct02/Sst02 parameters and POCD, and avoid the influence of collinearity among parameters, the Sct02/SstO2 parameters $(P<0.2$ in the univariable logistic regression analysis) were adjusted one by one using independent risk factors obtained in the final multivariate Logistic regression model. Statistical significance was set at $\mathrm{P}<0.05$.

\section{Results}

\section{Study population}

From December 2019 until April 2021, 112 patients scheduled for elective posterior lumbar or/and thoracic spine open surgery were included in this prospective observational study (Fig. 1). Eleven patients were excluded for data analysis. In three patients, there were no postoperative MMSE assessments, and 
in eight patients, there were more than $5 \%$ missing Sct02/Sst02 values. In the end, 101 patients were included in the data analysis. The postoperative MMSE positively correlated with preoperative MMSE $\left(\mathrm{r}^{2}\right.$ $=0.695, \mathrm{P}<0.001$, Fig. 2), while postoperative MMSE decreased, compared with preoperative MMSE $(24.9 \pm 2.9$ vs. $24.1 \pm 3.3, P<0.001$, Fig. 2 and Table 1$)$. No correlation was present between intraoperative Sct02 and intraoperative SstO2 $(P=0.702)$, nor was there any correlation between their respective absolute declines $(P=0.247)$ (Fig. 2). Of 101 patients, 28 (27.7\%) developed POCD. The number of 1, 2, 3 and 4 operative segments were performed in 62 (61.4\%), 34 (33.7\%), 4 (4.0\%) and 1 (1.0\%) patients, respectively (Table 2). Decompression and fusion were performed in $75(74.3 \%)$ and $74(73.3 \%)$ patients, respectively. The perioperative characteristic data in study population are shown in Table 1 and Table 2. 
Table 1

Preoperative characteristics in the study population, and comparison between the two groups

\begin{tabular}{|c|c|c|c|c|}
\hline Variables & $\begin{array}{l}\text { All patients }(\mathrm{n}= \\
101)\end{array}$ & $\operatorname{POCD}(n=28)$ & $\begin{array}{l}\text { No-POCD ( } \mathrm{n}= \\
73)\end{array}$ & $\begin{array}{l}\mathrm{P} \text { - } \\
\text { values }\end{array}$ \\
\hline \multicolumn{5}{|l|}{ Patient characteristics } \\
\hline Age (yr) & $64.8 \pm 9.3$ & $67.8 \pm 8.9$ & $63.7 \pm 9.3$ & 0.045 \\
\hline Male sex, n (\%) & $42(41.6)$ & $10(35.7)$ & $32(43.8)$ & 0.459 \\
\hline $\mathrm{BMI}\left(\mathrm{kg} \cdot \mathrm{m}^{-2}\right)$ & $24.7 \pm 3.2$ & $24.8 \pm 3.2$ & $24.6 \pm 3.2$ & 0.858 \\
\hline Current smoke, n (\%) & $30(29.7)$ & $8(28.6)$ & $22(30.1)$ & 0.878 \\
\hline Alcohol consumption, n (\%) & $21(20.8)$ & $4(14.3)$ & $17(23.3)$ & 0.318 \\
\hline ASA III, n (\%) & $47(46.5)$ & $19(67.9)$ & $28(38.4)$ & 0.008 \\
\hline ASA II, n (\%) & $54(53.5)$ & $9(32.1)$ & $45(61.6)$ & 0.008 \\
\hline \multicolumn{5}{|l|}{ Cognitive status } \\
\hline Education (years) & $6(4,11)$ & $6(4,11)$ & $6(4,11)$ & 0.818 \\
\hline Preoperative MMSE & $24.9 \pm 2.9$ & $24.6 \pm 3.2$ & $24.9 \pm 2.9$ & 0.647 \\
\hline Postoperative MMSE & $24.1 \pm 3.3$ & $21.8 \pm 3.6$ & $25.0 \pm 2.7$ & $<0.001$ \\
\hline \multicolumn{5}{|l|}{ Past medical history, $\mathrm{n}(\%)$} \\
\hline Hypertension & & & & 0.601 \\
\hline grade 1 & 40 (39.6) & $9(22.5)$ & $31(77.5)$ & \\
\hline grade 2 & 34 (33.7) & $10(29.4)$ & $24(70.6)$ & \\
\hline grade 3 & $27(26.7)$ & $9(33.3)$ & $18(66.7)$ & \\
\hline Coronary artery disease & $4(4.0)$ & $1(3.6)$ & $3(4.1)$ & 1.000 \\
\hline Previous stroke & $2(2.0)$ & 0 & $2(2.7)$ & 1.000 \\
\hline Diabetes mellitus & $23(22.8)$ & $6(21.4)$ & $17(23.3)$ & 0.842 \\
\hline COPD & $3(3.0)$ & $1(3.6)$ & $2(2.7)$ & 1.000 \\
\hline Chronic hepatitis B & $7(6.9)$ & $3(10.7)$ & $4(5.5)$ & 0.393 \\
\hline Chronic kidney disease & $9(8.9)$ & $5(17.9)$ & $4(5.5)$ & 0.111 \\
\hline
\end{tabular}

BMI, Body Mass Index; COPD, chronic obstructive pulmonary disease; ASA, American Society of Anesthesiologists; MMSE: Mini-Mental State Examination (MMSE) score; INR, International normalized ratio 


\begin{tabular}{|c|c|c|c|c|}
\hline Variables & $\begin{array}{l}\text { All patients }(n= \\
\text { 101) }\end{array}$ & $\operatorname{POCD}(n=28)$ & $\begin{array}{l}\text { No-POCD }(n= \\
73)\end{array}$ & $\begin{array}{l}\text { P- } \\
\text { values }\end{array}$ \\
\hline Hemoglobin (g/L) & $132 \pm 15$ & $132 \pm 14$ & $133 \pm 16$ & 0.786 \\
\hline White blood cell $\left(10^{9} / \mathrm{L}\right)$ & $6.5 \pm 1.9$ & $6.6 \pm 1.7$ & $6.5 \pm 2.0$ & 0.841 \\
\hline Platelet $\left(10^{9} / \mathrm{L}\right)$ & $167 \pm 56$ & $194 \pm 68$ & $157 \pm 47$ & 0.002 \\
\hline INR & $1.05 \pm 0.07$ & $1.04 \pm 0.09$ & $1.05 \pm 0.06$ & 0.532 \\
\hline Alanine transaminase $(U)$ & $21.2(16.8,29.6)$ & $\begin{array}{l}20.6 \\
(15.4,37.4)\end{array}$ & $22.0(16.8,28.2)$ & 0.900 \\
\hline $\begin{array}{l}\text { Aspartate aminotransferase } \\
(\mathrm{U})\end{array}$ & $23.1(18.2,29.4)$ & $\begin{array}{l}24.3 \\
(17.8,29.2)\end{array}$ & $22.8(18.4,29.7)$ & 0.903 \\
\hline Albumin (g/L) & $41.0 \pm 3.7$ & $40.5 \pm 3.9$ & $41.2 \pm 3.7$ & 0.444 \\
\hline $\begin{array}{l}\text { Glomerular filtration rate } \\
(\mathrm{ml} / \mathrm{min})\end{array}$ & $99(84,121)$ & $99(85,115)$ & $99(82,126)$ & 0.497 \\
\hline \multicolumn{5}{|c|}{$\begin{array}{l}\text { BMI, Body Mass Index; COPD, chronic obstructive pulmonary disease; ASA, American Society of } \\
\text { Anesthesiologists; MMSE: Mini-Mental State Examination (MMSE) score; INR, International } \\
\text { normalized ratio }\end{array}$} \\
\hline
\end{tabular}


Table 2

Intraoperative and postoperative characteristics in the study population, and comparison between the two groups

\begin{tabular}{|c|c|c|c|c|}
\hline Variables & All patients & POCD & No-POCD & $\begin{array}{l}\mathrm{P} \text { - } \\
\text { values }\end{array}$ \\
\hline \multicolumn{5}{|l|}{ Surgical information } \\
\hline Number of segments (n) & & & & 0.843 \\
\hline 1 & $62(61.4)$ & $19(67.9)$ & $43(58.9)$ & \\
\hline 2 & $34(33.7)$ & $8(28.6)$ & $26(35.6)$ & \\
\hline 3 & $4(4.0)$ & $1(3.6)$ & $3(4.1)$ & \\
\hline 4 & $1(1.0)$ & 0 & $1(1.4)$ & \\
\hline Decompression, n (\%) & $75(74.3)$ & $24(85.7)$ & $51(69.9)$ & 0.103 \\
\hline Fusion, n (\%) & $74(73.3)$ & $23(82.1)$ & $51(69.9)$ & 0.212 \\
\hline Surgical time (minutes) & $165(140,197)$ & $173(141,201)$ & $164(137,195)$ & 0.464 \\
\hline \multicolumn{5}{|l|}{ Intraoperative management } \\
\hline Fluid infusion volume (ml) & $\begin{array}{l}1700 \\
(1300,2000)\end{array}$ & $\begin{array}{l}1650 \\
(1250,2150)\end{array}$ & $\begin{array}{l}1700 \\
(1300,2000)\end{array}$ & 0.967 \\
\hline Colloid (ml) & $500(0,500)$ & $500(0,500)$ & $500(0,500)$ & 0.418 \\
\hline Transfusion, n (\%) & $2(2.0)$ & $2(7.1)$ & 0 & 0.075 \\
\hline Estimated blood loss (ml) & $300(200,500)$ & $325(200,500)$ & $300(200,525)$ & 0.464 \\
\hline Urine output (ml)* & $250(150,400)$ & $200(135,450)$ & $250(200,300)$ & 0.665 \\
\hline Lactate when suturing $(\mathrm{mmol} / \mathrm{L})$ & $1.39(1.08,1.75)$ & $1.42(1.15,1.79)$ & $1.39(1.07,1.74)$ & 0.741 \\
\hline Ephedrine (mg) & $12(6,18)$ & $12(3,18)$ & $12(6,18)$ & 0.458 \\
\hline Ephedrine (n (\%)) & $82(81.2)$ & $21(75.0)$ & $61(83.6)$ & 0.324 \\
\hline Phenylephrine (ug) & $0(0,0)$ & $0(0,219)$ & $0(0,0)$ & 0.013 \\
\hline Phenylephrine (n (\%)) & $21(20.8)$ & $10(35.7)$ & $11(15.1)$ & 0.022 \\
\hline \multicolumn{5}{|l|}{ Postoperative management } \\
\hline Extubation time (minutes) & $21(15,33)$ & $21(15,34)$ & $23(15,33)$ & 0.761 \\
\hline Length of PACU stay (minutes) & $50(40,67)$ & $51(40,67)$ & $50(40,67)$ & 0.764 \\
\hline Self-controlled analgesia, n (\%) & $89(88.1)$ & $25(89.3)$ & $64(87.7)$ & 0.822 \\
\hline
\end{tabular}




\begin{tabular}{|c|c|c|c|c|}
\hline Variables & All patients & POCD & No-POCD & $\begin{array}{l}\mathrm{P} \text { - } \\
\text { values }\end{array}$ \\
\hline NPS in postoperative $1 \mathrm{~d}$ (points) & $1(1,2)$ & $1(1,2)$ & $1(1,2)$ & 0.358 \\
\hline NPS in postoperative $3 \mathrm{~d}$ (points) & $1(1,1)$ & $1(1,2)$ & $1(1,1)$ & 0.127 \\
\hline $\begin{array}{l}\text { Postoperative } 24 \mathrm{~h} \text { drainage } \\
\text { volume (ml) }\end{array}$ & $150(53,227)$ & $155(80,298)$ & $100(23,205)$ & 0.044 \\
\hline Length of hospital stay (days) & $14(10,20)$ & $15(12,19)$ & $14(10,20)$ & 0.352 \\
\hline $\begin{array}{l}\text { Length of postoperative hospital } \\
\text { stay (days) }\end{array}$ & $10(7,14)$ & $11(7,16)$ & $10(7,14)$ & 0.367 \\
\hline Total medical costs (RMB) & $\begin{array}{l}48851 \\
(40118,58028)\end{array}$ & $\begin{array}{l}50250 \\
(40015,64673)\end{array}$ & $\begin{array}{l}48779 \\
(40118,55876)\end{array}$ & 0.421 \\
\hline \multicolumn{5}{|l|}{ Postoperative complications } \\
\hline Cerebrospinal leak, n (\%) & $8(7.9)$ & $5(17.9)$ & $3(4.1)$ & 0.036 \\
\hline Postoperative sepsis, n (\%) & $9(8.9)$ & $5(17.9)$ & $4(5.5)$ & 0.111 \\
\hline $\begin{array}{l}\text { Thrombocytopenia (Platelet < } \\
\text { 100,000), n (\%) }\end{array}$ & $16(15.8)$ & $5(17.9)$ & $11(15.1)$ & 0.765 \\
\hline \multicolumn{5}{|c|}{$\begin{array}{l}\text { *, Only } 64 \text { were included because the remaining } 47 \text { did not receive intraoperative catheterization; } \\
\text { PACU, post-anesthesia care unit; NPS, numerical pain score; RMB, Renminbi }\end{array}$} \\
\hline
\end{tabular}

The preoperative characteristic data of both patient groups are shown in Table 1. The patients with POCD were older, had a higher rate of chronic kidney disease and ASA III, and had a higher number of platelet compared with the patients without POCD. The length of education and the preoperative MMSE scores were similar in both groups $(P=0.818, P=0.647$; respectively), while the patients with POCD scored lower than the ones without POCD on the postoperative MMSE $(P<0.001$; Table 1). Perioperative management and postoperative complications data of both groups are shown in Table 2. POCD patients had a higher proportion of intraoperative phenylephrine use, more postoperative $24 \mathrm{~h}$ drainage, and a higher proportion of postoperative cerebrospinal leak. However, there was no difference in intraoperative blood loss and proportion of intraoperative ephedrine use between two groups.

\section{Comparison of Sct02 and Sst02 between two groups}

The univariable comparison of intraoperative NIRS (both Sct02 and Sst02) parameters between the patients with and without POCD is shown in Table 3. The baseline SctO2, lowest intraoperative SctO2, mean Sct02 and median Sct02 were similar between the patients with and without POCD $(P=0.593$, $0.947,0.728$ and 0.722 , respectively). Likewise, intraoperative absolute SctO2 decrease, relative Sct02 decrease, the AUC values of postoperative Sct02 values below $60 \%, 55 \%$ and $50 \%$, the times below $80 \%$ of baseline SctO2 and the times below $90 \%$ of baseline SctO2 did not differ between both groups ( $\mathrm{P}=$ 
$0.649,0.730,0.296,0.239,0.201,0.682$ and 0.561 , respectively). Generally, none of the investigated SctO2 variables was predictive of POCD. In contrast to SctO2 measured from the forehead, the patients with POCD had a higher decrease in intraoperative absolute SstO2 decrease and relative SstO2 decrease compared with the patients without POCD $(4.9 \% \pm 3.8 \%$ vs. $3.6 \% \pm 2.6 \%, \mathrm{P}=0.037 ; 7.4 \% \pm 5.6 \%$ vs. $5.3 \%$ $\pm 3.8 \%, P=0.036$; respectively; Fig. 3). The proportion below $90 \%$ of baseline Sst02 in the patients with POCD were significantly higher than patients without $(32.1 \%$ vs. $5.5 \%, \mathrm{P}<0.001)$, but $19(67.9 \%)$ of the 28 patients who developed POCD did not experience intraoperative SstO2 desaturations below $90 \%$ of baseline (Fig. 3). Unfortunately, the times below $80 \%$ of baseline Sst02 did not differ between both groups, as the frequency of below $80 \%$ baseline Sct02 was 2 , in 1 case in each group (Table 1). In addition, other investigated Sst02 variables such as baseline Sst02, lowest intraoperative Sst02, mean SstO2 and median SstO2 did not differ significantly between both groups (Table 1). 
Table 3

Intraoperative cerebral and somatic tissue oxygenation in the study population, and comparison between the two groups

\begin{tabular}{|c|c|c|c|c|}
\hline Variables & All patients & $\operatorname{POCD}(n=28)$ & $\begin{array}{l}\text { No-POCD }(n= \\
73)\end{array}$ & $\begin{array}{l}\mathrm{P}- \\
\text { values }\end{array}$ \\
\hline \multicolumn{5}{|l|}{$\begin{array}{l}\text { Cerebral tissue oxygen saturation } \\
\text { (Sct02) }\end{array}$} \\
\hline Baseline SctO2 (\%) & $64.2 \pm 3.9$ & $64.6 \pm 4.8$ & $64.1 \pm 3.5$ & 0.593 \\
\hline Lowest SctO2 (\%) & $56.9 \pm 3.9$ & $56.9 \pm 4.8$ & $56.9 \pm 3.6$ & 0.947 \\
\hline Sct02 mean (\%) & $62.1 \pm 3.7$ & $62.3 \pm 4.3$ & $62.0 \pm 3.5$ & 0.728 \\
\hline SctO2 median (\%) & $61.5 \pm 3.9$ & $61.7 \pm 4.4$ & $61.4 \pm 3.8$ & 0.722 \\
\hline Absolute SctO2 decrease (\%) & $7.4 \pm 4.0$ & $7.6 \pm 4.8$ & $7.2 \pm 3.6$ & 0.649 \\
\hline Relative Sct02 decrease (\%) & $11.3+5.8$ & $11.6 \pm 7.0$ & $11.2 \pm 5.3$ & 0.730 \\
\hline Sct02 AUC 60\% (\%*min) & $17(0,235)$ & $5(0,212)$ & $27(0,226)$ & 0.296 \\
\hline SctO2 AUC 55\% (\%*min) & $0(0,0)$ & $0(0,2)$ & $0(0,0)$ & 0.239 \\
\hline SctO2 AUC 50\% (\%*min) & $0(0,0)$ & $0(0,0)$ & $0(0,0)$ & 0.201 \\
\hline Desaturation $<80 \%$ of baseline, $\mathrm{n}(\%)$ & $8(7.9)$ & $3(10.7)$ & $5(6.8)$ & 0.682 \\
\hline Desaturation < $90 \%$ of baseline, $\mathrm{n}(\%)$ & $53(52.5)$ & $16(57.1)$ & $37(50.7)$ & 0.561 \\
\hline \multicolumn{5}{|l|}{$\begin{array}{l}\text { Somatic tissue oxygen saturation } \\
\text { (Sst02) }\end{array}$} \\
\hline Baseline SstO2 (\%) & $\begin{array}{l}68.3 \\
(64.9,70.3)\end{array}$ & $\begin{array}{l}67.8 \\
(64.6,70.0)\end{array}$ & $\begin{array}{l}68.4 \\
(64.9,70.4)\end{array}$ & 0.682 \\
\hline Lowest SstO2 (\%) & $\begin{array}{l}64.0 \\
(61.0,66.4)\end{array}$ & $\begin{array}{l}62.6 \\
(60.6,65.2)\end{array}$ & $\begin{array}{l}64.5 \\
(61.4,66.5)\end{array}$ & 0.088 \\
\hline SstO2 mean (\%) & $\begin{array}{l}68.7 \\
(66.2,70.5)\end{array}$ & $\begin{array}{l}68.0 \\
(65.8,70.0)\end{array}$ & $\begin{array}{l}68.7 \\
(67.1,70.6)\end{array}$ & 0.205 \\
\hline Sst02 median (\%) & $\begin{array}{l}68.8 \\
(66.270 .6)\end{array}$ & $\begin{array}{l}67.8 \\
(65.7,69.8)\end{array}$ & $\begin{array}{l}68.9 \\
(67.2,70.6)\end{array}$ & 0.176 \\
\hline Absolute SstO2 decrease (\%) & $3.9 \pm 3.0$ & $4.9 \pm 3.8$ & $3.6 \pm 2.6$ & 0.037 \\
\hline Relative SstO2 decrease (\%) & $5.9 \pm 4.4$ & $7.4 \pm 5.6$ & $5.3 \pm 3.8$ & 0.036 \\
\hline SstO2 AUC 60\% (\%*min) & $0(0,0)$ & $0(0,0)$ & $0(0,0)$ & 0.933 \\
\hline SstO2 AUC 55\% (\%*min) & $0(0,0)$ & $0(0,0)$ & $0(0,0)$ & 0.237 \\
\hline SstO2 AUC 50\% (\%*min) & - & - & - & - \\
\hline
\end{tabular}

AUC, area under the curve 


\begin{tabular}{|lllll|}
\hline Variables & All patients & POCD $(n=28)$ & $\begin{array}{l}\text { No-POCD }(n= \\
73)\end{array}$ & $\begin{array}{l}\text { P- } \\
\text { values }\end{array}$ \\
\hline Desaturation $<80 \%$ of baseline, $n(\%)$ & $2(2.0)$ & $1(3.6)$ & $1(1.4)$ & 0.480 \\
\hline Desaturation < $90 \%$ of baseline, $n(\%)$ & $13(12.9)$ & $9(32.1)$ & $4(5.5)$ & 0.001 \\
\hline AUC, area under the curve & & & & \\
\hline
\end{tabular}

\section{Association between Sct02/Sst02 and POCD}

The suspicious risk factors about POCD were variables with $\mathrm{P}<0.2$ identified by conducting univariate logistic regression, including age, ASA III, education level, preoperative chronic kidney disease, preoperative platelet, intraoperative decompression, intraoperative phenylephrine, postoperative cerebrospinal leak, postoperative $24 \mathrm{~h}$ drainage volume, postoperative sepsis and numerical pain score in postoperative 3d (Supplementary Table 1). Finally, three SstO2 parameters respectively were associated with POCD, including a higher absolute SstO2 decrease (OR, 1.223; 95\% Cl, 1.031-1.451; $\mathrm{P}=0.021)$, a higher absolute SstO2 decrease $(\mathrm{OR}, 1.138 ; 95 \% \mathrm{Cl}, 1.011-1.281 ; \mathrm{P}=0.032)$ and falling below $90 \%$ of baseline SstO2 (OR, 11.388; 95\% Cl, 2.367-54.785; $\mathrm{P}=0.002)$, independent of ASA III, preoperative platelet and postoperative sepsis (Table 4). Likewise, A negative correlation was present between the parameters of Sct02 (e.g., the absolute decrease) and the length of postoperative hospital stay $\left(r^{2}=0.082, P=0.004\right.$, Fig. 3), while the parameters of SctO2 were not. 
Table 4

Univariable and multivariable logistic regression analysis of the Sct02/Sst02 parameters for prediction of POCD

\begin{tabular}{|c|c|c|c|c|c|c|}
\hline \multirow[t]{2}{*}{ Variables } & \multicolumn{3}{|c|}{ Univariable } & \multicolumn{3}{|c|}{ Multivariable } \\
\hline & OR & $95 \% \mathrm{Cl}$ & $\begin{array}{l}\mathrm{P} \text { - } \\
\text { values }\end{array}$ & OR & $95 \% \mathrm{Cl}$ & $\begin{array}{l}\text { P- } \\
\text { values }\end{array}$ \\
\hline ASA III (vs. ASA II) & 3.393 & $(1.348,8.538)$ & 0.009 & 3.992 & $(1.434,11.112)$ & 0.008 \\
\hline Platelet, per $10^{9} / \mathrm{L}$ & 1.012 & $(1.004,1.021)$ & 0.005 & 1.012 & $(1.003,1.021)$ & 0.009 \\
\hline Postoperative sepsis & 3.750 & $(0.928,15.160)$ & 0.064 & 5.201 & $(1.057,25.583)$ & 0.042 \\
\hline \multicolumn{7}{|l|}{$\begin{array}{l}\text { Cerebral tissue } \\
\text { oxygen saturation } \\
\text { (Sct02) }\end{array}$} \\
\hline Baseline Sct02, per \% & 1.032 & $(0.921,1.155)$ & 0.589 & & & \\
\hline Lowest Sct02, per \% & 1.004 & $(0.898,1.122)$ & 0.947 & & & \\
\hline $\begin{array}{l}\text { Absolute SctO2 } \\
\text { decrease, per \% }\end{array}$ & 1.026 & $(0.920,1.145)$ & 0.645 & & & \\
\hline $\begin{array}{l}\text { Relative Sct02 } \\
\text { decrease, per \% }\end{array}$ & 1.014 & $(0.940,1.093)$ & 0.727 & & & \\
\hline SctO2 mean, per \% & 1.021 & $(0.908,1.150)$ & 0.725 & & & \\
\hline Sct02 median, per \% & 1.021 & $(0.913,1.142)$ & 0.719 & & & \\
\hline $\begin{array}{l}\text { SctO2 AUC } 60 \% \text {, per } \\
\% \star \min \end{array}$ & 1.000 & $(0.999,1.001)$ & 0.624 & & & \\
\hline $\begin{array}{l}\text { SctO2 AUC 55\%, per } \\
\%^{*} \min \end{array}$ & 1.002 & $(0.998,1.005)$ & 0.314 & & & \\
\hline $\begin{array}{l}\text { SctO2 AUC 50\%, per } \\
\%^{*} \text { min }\end{array}$ & 0.994 & $(0.961,1.029)$ & 0.747 & & & \\
\hline $\begin{array}{l}\text { Desaturation }<80 \% \text { of } \\
\text { baseline }\end{array}$ & 1.632 & $(0.363,7.336)$ & 0.523 & & & \\
\hline $\begin{array}{l}\text { Desaturation }<90 \% \text { of } \\
\text { baseline }\end{array}$ & 1.297 & $(0.539,3.121)$ & 0.561 & & & \\
\hline \multicolumn{7}{|l|}{$\begin{array}{l}\text { Somatic tissue } \\
\text { oxygen saturation } \\
\text { (Sct02) }\end{array}$} \\
\hline Baseline Sct02, per \% & 0.992 & $(0.891,1.105)$ & 0.883 & & & \\
\hline
\end{tabular}

OR, odds ratio; $\mathrm{Cl}$, confidence interval; ASA, American Society of Anesthesiologists; AUC, area under the curve 


\begin{tabular}{|c|c|c|c|c|c|c|}
\hline \multirow{2}{*}{$\begin{array}{l}\text { Variables } \\
\text { SctO2 minimum, per } \\
\%\end{array}$} & \multicolumn{3}{|c|}{ Univariable } & \multicolumn{3}{|c|}{ Multivariable } \\
\hline & 0.939 & $(0.859,1.026)$ & 0.162 & 0.969 & $(0.871,1.077)$ & 0.558 \\
\hline SctO2 mean, per \% & 0.921 & $(0.818,1.037)$ & 0.174 & 0.934 & $(0.811,1.076)$ & 0.346 \\
\hline Sct02 median, per $\%$ & 0.919 & $(0.818,1.032)$ & 0.153 & 0.922 & $(0.802,1.060)$ & 0.256 \\
\hline $\begin{array}{l}\text { Absolute SctO2 } \\
\text { decrease, per \% }\end{array}$ & 1.159 & $(1.003,1.339)$ & 0.046 & 1.223 & $(1.031,1.451)$ & 0.021 \\
\hline $\begin{array}{l}\text { Relative SctO2 } \\
\text { decrease, per \% }\end{array}$ & 1.105 & $(1.003,1.218)$ & 0.044 & 1.138 & $(1.011,1.281)$ & 0.032 \\
\hline $\begin{array}{l}\text { Sct02 AUC60, per } \\
\% * \min \end{array}$ & 1.002 & $(0.999,1.006)$ & 0.166 & 1.003 & $(0.998,1.007)$ & 0.260 \\
\hline $\begin{array}{l}\text { SctO2 AUC55, per } \\
\text { \%*min }\end{array}$ & 1.040 & $(0.917,1.179)$ & 0.546 & & & \\
\hline $\begin{array}{l}\text { Sct02 AUC50, per } \\
\% * \text { min }\end{array}$ & - & - & - & & & \\
\hline $\begin{array}{l}\text { Desaturation }<80 \% \text { of } \\
\text { baseline }\end{array}$ & 2.667 & $(0.161,44.155)$ & 0.493 & & & \\
\hline $\begin{array}{l}\text { Desaturation }<90 \% \text { of } \\
\text { baseline }\end{array}$ & 8.171 & $(2.266,29.465)$ & 0.001 & 11.388 & $(2.367,54.785)$ & 0.002 \\
\hline \multicolumn{7}{|c|}{$\begin{array}{l}\text { OR, odds ratio; } \mathrm{Cl} \text {, confidence interval; ASA, American Society of Anesthesiologists; AUC, area under } \\
\text { the curve }\end{array}$} \\
\hline
\end{tabular}

\section{Discussion}

In this prospective observational study, 28 (27.7\%) of 101 patients developed POCD, which was slightly higher than the result (23\%) in the study of Jeongmin Kim on spinal surgery. ${ }^{[11]}$ This may be because Jeongmin Kim's study was on elderly patients, while our study was on patients with hypertension in whom the average age was 64.8. Hypertension can raise the brain's threshold for self-regulation of blood pressure, cause blood-brain barrier breakdown, and frequently encounter labile hemodynamics during surgery, which is more likely to lead to cognitive decline. ${ }^{[1,20,21]}$ The results of this study showed that POCD was strongly associated with the absolute decrease in intraoperative SstO2, but had little correlation with $\mathrm{rScO} 2$, in patients with hypertension undergoing open posterior spine surgery.

As the brain is known to consume a lot of oxygen that is supplied to the body, the cerebral function is very sensitive and vulnerable to hypoxemia. In the setting of open posterior spine surgery, the importance of avoiding cerebral oxygen deficiency has been increasingly recognized, and cerebral oximetry, as a noninvasive tool to assess the adequacy of cerebral oxygen delivery, is increasingly used in the clinic. However, there were few studies to monitor cerebral oxygen saturation and somatic oxygen saturation 
simultaneously. The results of this study showed that there was no correlation between intraoperative cerebral oxygen saturation and somatic oxygen saturation, nor was there any correlation between their respective declines. These results may suggest that there may be significant differences in the correlation between the oxygen saturation of different sites and the clinical outcome and prognosis. The strategy of multi-site monitoring may be beneficial at present, and identifying the best monitoring site is important to further improve patient outcomes. ${ }^{[22]}$

In the clinic, the purpose of monitoring is to simply and effectively prevent and reduce the occurrence of postoperative complications. So far, there have been few comparisons of the correlation between different sites monitoring tissue oxygen and POCD, and it is not clear which site is better. In this study, there was no correlation between any indicator of intraoperative SctO2 in the forehead and POCD. This result was similar with the result of previous study that they found no correlation between intraoperative SctO2 and postoperative complications after spine surgery. ${ }^{[17]}$ But, this result was completely inconsistent with other studies showing a good correlation between SctO2 and POCD in non-cardiac surgery. Although many preventive measures had been taken, the specificity of prone position might still affect the measurement of SctO2 in this study for two reasons: First, all patients were in prone position, which might result in cephalic and facial venous pooling. Second, patients in a prone position often have edema formation in the lower part of the body, including the forehead. Therefore, the prevention of neurological impairment by cerebral oxygen saturation may be seriously interfered with and weakened undergoing surgery in a prone position. Unfortunately, recent findings in prone surgery are conflicting, and postural factors may not be able to explain all causes. ${ }^{[11,17]}$ Further studies are needed to clarify the relationship between intraoperative SctO2 and postoperative complications in prone surgery.

In contrast, the present study found that intraoperative somatic tissue desaturations during spine surgery were significantly associated with POCD. Thus, we proved the previous hypothesis that SctO2 in the forehead is affected by position in prone position, while SstO2 is not. This also implied a potentially modifiable causal link, thereby concluding that a persisting somatic desaturation should be avoided. This result is similar to that of Meng $L$ et al. ${ }^{[17]}$ They found that muscular tissue desaturations were significantly associated with postoperative complications. However, in this study, the monitoring site was the midaxillary line of the waist at the same height as the operation, while in their study, the lower leg was monitored. The main reasons why we did not choose the lower leg and the more common arm (especially the thenar muscle) for SstO2 measurement are as follows: First, bilateral thenar muscles were usually used for evoked potential monitoring in our patients. Second, the monitoring locations we selected were almost at the same level as the vital organs and the brain, where hemodynamics might be more similarly affected by relative position. Third, the sites we monitored may involve muscle tissue or even splanchnic organs such as small intestine, colon or liver that are more sensitive to ischemia and hypoxia. Nearly all splanchnic organs possess weak autoregulation than the brain and heart. ${ }^{[23]}$ Intuitively, during periods of hemodynamic instability, sites with the lowest priority may have a better correlation with postoperative complications. Therefore, our results further confirmed that different monitoring sites of local tissue oxygen saturation have different effects on the same outcome index, and it is important to study the best 
monitoring sites of specific prognostic index and its specific clinical practical value in the future. Unfortunately, even among the intraoperative Sst02 variables we studied, none completely distinguished POCD patients from non-POCD patients. Correspondingly, neither Sct02 nor SstO2 measured preoperatively was predictive for $P O C D$, in contrast to previous reports. In addition, our study found that SstO2 desaturation did not occur in some POCD patients.

This current study indicated that the patients with POCD were more likely to have lower Sst02 values during surgery, and more likely to experience somatic desaturations below $90 \%$ of preoperative baseline. What's more, a higher absolute/relative decrease in postoperative Sst02 was associated with POCD, independent of other risk factors, such as ASA III, preoperative platelet and postoperative sepsis.

This study had at least six limitations as follows: First, this was a single-center study with a small sample size, and the sample size was not pre-determined before the study. Hence, there is a chance that the association of intraoperative somatic desaturation and cerebral desaturation with POCD after spine surgery may be underestimated. Larger multicenter studies are needed to confirm these associations. Second, this was an observational study, and therefore the somatic desaturation might be a manifestation or consequence of POCD rather than a causal mediator thereof. Third, different NIRS monitors are commercially available, which allow monitoring tissue oxygenation either cerebrally or peripherally. The differences in the methodological approaches may lead to the bias and variability between different devices. ${ }^{[24]}$ Therefore, study results obtained with different NIRS devices might be hardly applicable and comparable with each other, which might partially explain the conflicting of the results from published studies. Fourth, the patients with hypertension included in this study were usually elderly, and the elderly generally had low education due to historical reasons (Table 1). Since education level may have a great influence on MMSE, the preoperative MMSE score in this study was relatively low. To ensure the representativeness of the study, we excluded the patients with preoperative MMSE $<18$ rather than preoperative MMSE < 24 (Fig. 1). Fifth, as postoperative MMSE assessment was conducted only on the fourth day after surgery, it was not possible to determine the exact point in time when the patients developed POCD. This may lead to an underestimation of the incidence of cognitive dysfunction after spine surgery in patients with hypertension. Sixth, other studies were also using NIRS devices, so not all eligible patients were included. This could have increased the selection bias of the study.

\section{Conclusion And Outlook}

In summary, POCD can occur in $28 \%$ of patients with hypertension undergoing open posterior spine surgery. Intraoperative somatic tissue oxygen saturation has an association with POCD after surgery, while cerebral tissue oxygenation has little association with POCD in this patient population. At present, a combination of both cerebral and somatic tissue oxygenation monitoring might therefore offer a more complete assessment of the mismatches between oxygen supply and consumption. It is necessary to further clarify the best monitoring locations, the sensitive monitoring indicators, and their relationship to clinical outcomes, especially in high-risk patients undergoing high-risk non-cardiac surgery, for which few studies have been conducted. 


\section{Abbreviations}

Sct02,regional cerebral oxygen saturation; Sst02,somatic tissue oxygen saturation; ASA, American Society of Anesthesiologists; MMSE, mini-mental state examination; NIRS, Near-infrared spectroscopy; BMI, Body Mass Index; COPD, chronic obstructive pulmonary disease; INR, International normalized ratio; PACU, post-anesthesia care unit; NPS, numerical pain score; RMB, Renminbi; AUC, area under the curve; OR, odds ratio; $\mathrm{Cl}$, confidence interval; $\mathrm{ROC}$, receiver operating characteristic

\section{Declarations}

\section{Acknowledgements}

Not applicable.

\section{Funding}

This study was supported by grants from the Hypertension Special Research Project (SHI HUI DA) of Sichuan Medical Association (Grant No.2018SHD1-10), and Key Science and Technology Program of Zigong City, Sichuan Province(Grant No.2018CZ15).

\section{Availability of data and materials}

The data that support the findings of this study are available from the corresponding author upon reasonable request.

\section{Author contributions}

FG, YYZ, SYJ and BL were responsible for the conception and design of the study. FG, QYW, WZW,MQH and SYJ were responsible for the acquisition and analysis of the data. FG, YYZ, SJL and QL were responsible for the interpretation of the data and drafted the manuscript. All authors agree to be accountable for all aspects of the work in ensuring that questions related to the accuracy or integrity of any part of the work are appropriately investigated and resolved. All authors read and approved the final manuscript.

\section{Ethics approval and consent to participate}

This study was approved by Zigong Fourth People's Hospital Affiliated to Southwest Medical University ethics committee, ethical approval (2020KY024), and Chinese clinical trial registry (registration number: ChiCTR1900028392), Written informed consent was obtained from all patients before inclusion.

\section{Competing interests}

The authors declare that they have no competing interests. 


\section{Consent for publication}

Not applicable.

\section{References}

1. Yocum GT, Gaudet JG, Teverbaugh LA, et al. Neurocognitive performance in hypertensive patients after spine surgery. Anesthesiology. 2009;110(2):254-61.

2. Van Dijk D. Long-term cognitive impairment after critical illness. N Engl J Med. 2014;370(2):185.

3. Steinmetz J, Rasmussen LS. Peri-operative cognitive dysfunction and protection. Anaesthesia. 2016;71(Suppl 1):58-63.

4. Newman MF, Kirchner JL, Phillips-Bute B, et al. Longitudinal assessment of neurocognitive function after coronary-artery bypass surgery. N Engl J Med. 2001;344(6):395-402.

5. Evered LA, Silbert BS. Postoperative Cognitive Dysfunction and Noncardiac Surgery. Anesth Analg. 2018;127(2):496-505.

6. Kotekar N, Shenkar A, Nagaraj R. Postoperative cognitive dysfunction - current preventive strategies. Clin Interv Aging. 2018;13:2267-73.

7. Ikeda K, MacLeod DB, Grocott HP, Moretti EW, Ames W, Vacchiano C. The accuracy of a near-infrared spectroscopy cerebral oximetry device and its potential value for estimating jugular venous oxygen saturation. Anesth Analg. 2014;119(6):1381-92.

8. Mu DL, Wang DX, Meng L. Incremental value of noncerebral somatic tissue oxygenation monitoring for patients undergoing surgery. Curr Opin Anaesthesiol. 2019;32(1):50-6.

9. Vos JJ, Ellermann SF, Scheeren T. Journal of Clinical Monitoring and Computing 2017/2018 end of year summary: monitoring-and provocation-of the microcirculation and tissue oxygenation. J Clin Monit Comput. 2019;33(2):201-9.

10. Lin L, Li G, Li J, Meng L. Tourniquet-induced tissue hypoxia characterized by near-infrared spectroscopy during ankle surgery: an observational study. BMC Anesthesiol. 2019;19(1):70.

11. Kim J, Shim JK, Song JW, Kim EK, Kwak YL. Postoperative Cognitive Dysfunction and the Change of Regional Cerebral Oxygen Saturation in Elderly Patients Undergoing Spinal Surgery. Anesth Analg. 2016;123(2):436-44.

12. Carra G, Flechet $M$, Jacobs A, et al. Postoperative Cerebral Oxygen Saturation in Children After Congenital Cardiac Surgery and Long-Term Total Intelligence Quotient: A Prospective Observational Study. Crit Care Med. 2021;49(6):967-76.

13. Eertmans W, De Deyne C, Genbrugge C, et al. Association between postoperative delirium and postoperative cerebral oxygen desaturation in older patients after cardiac surgery. $\mathrm{Br} \mathrm{J}$ Anaesth. 2020;124(2):146-53.

14. Agbangla NF, Audiffren M, Albinet CT. Use of near-infrared spectroscopy in the investigation of brain activation during cognitive aging: A systematic review of an emerging area of research. Ageing Res 
Rev. 2017;38:52-66.

15. Cui F, Zhao W, Mu DL, et al. Association Between Cerebral Desaturation and Postoperative Delirium in Thoracotomy With One-Lung Ventilation: A Prospective Cohort Study. Anesth Analg. 2021;133(1):176-86.

16. Sandroni C, Parnia S, Nolan JP. Cerebral oximetry in cardiac arrest: a potential role but with limitations. Intensive Care Med. 2019;45(6):904-6.

17. Meng L, Xiao J, Gudelunas K, Yu Z, Zhong Z, Hu X. Association of intraoperative cerebral and muscular tissue oxygen saturation with postoperative complications and length of hospital stay after major spine surgery: an observational study. Br J Anaesth. 2017;118(4):551-62.

18. Arevalo-Rodriguez I, Smailagic N, Roqué I, Figuls M, et al. Mini-Mental State Examination (MMSE) for the detection of Alzheimer's disease and other dementias in people with mild cognitive impairment (MCl). Cochrane Database Syst Rev. 2015. (3): CD010783.

19. Burdick DJ, Cholerton B, Watson GS, et al. People with Parkinson's disease and normal MMSE score have a broad range of cognitive performance. Mov Disord. 2014;29(10):1258-64.

20. Pires PW, Dams Ramos CM, Matin N, Dorrance AM. The effects of hypertension on the cerebral circulation. Am J Physiol Heart Circ Physiol. 2013;304(12):H1598-614.

21. Levin MA, Fischer GW, Lin HM, McCormick PJ, Krol M, Reich DL. Intraoperative arterial blood pressure lability is associated with improved 30 day survival. Br J Anaesth. 2015;115(5):716-26.

22. Fan X, Lin L, Li G, et al. Do cerebral and somatic tissue oxygen saturation measurements correlate with each other during surgery. J Clin Monit Comput. 2020;34(3):483-90.

23. Meng L, Wang Y, Zhang L, McDonagh DL. Heterogeneity and Variability in Pressure Autoregulation of Organ Blood Flow: Lessons Learned Over 100 + Years. Crit Care Med. 2019;47(3):436-48.

24. Thavasothy M, Broadhead M, Elwell C, Peters M, Smith M. A comparison of cerebral oxygenation as measured by the NIRO 300 and the INVOS 5100 Near-Infrared Spectrophotometers. Anaesthesia. 2002;57(10):999-1006.

25. Lee JH, Park YH, Kim HS, Kim JT. Comparison of two devices using near-infrared spectroscopy for the measurement of tissue oxygenation during a vascular occlusion test in healthy volunteers (INVOS ${ }^{\circledR}$ vs. InSpectra ${ }^{\mathrm{TM}}$ ). J Clin Monit Comput. 2015;29(2):271-8.

\section{Figures}




\section{9 patients screened}

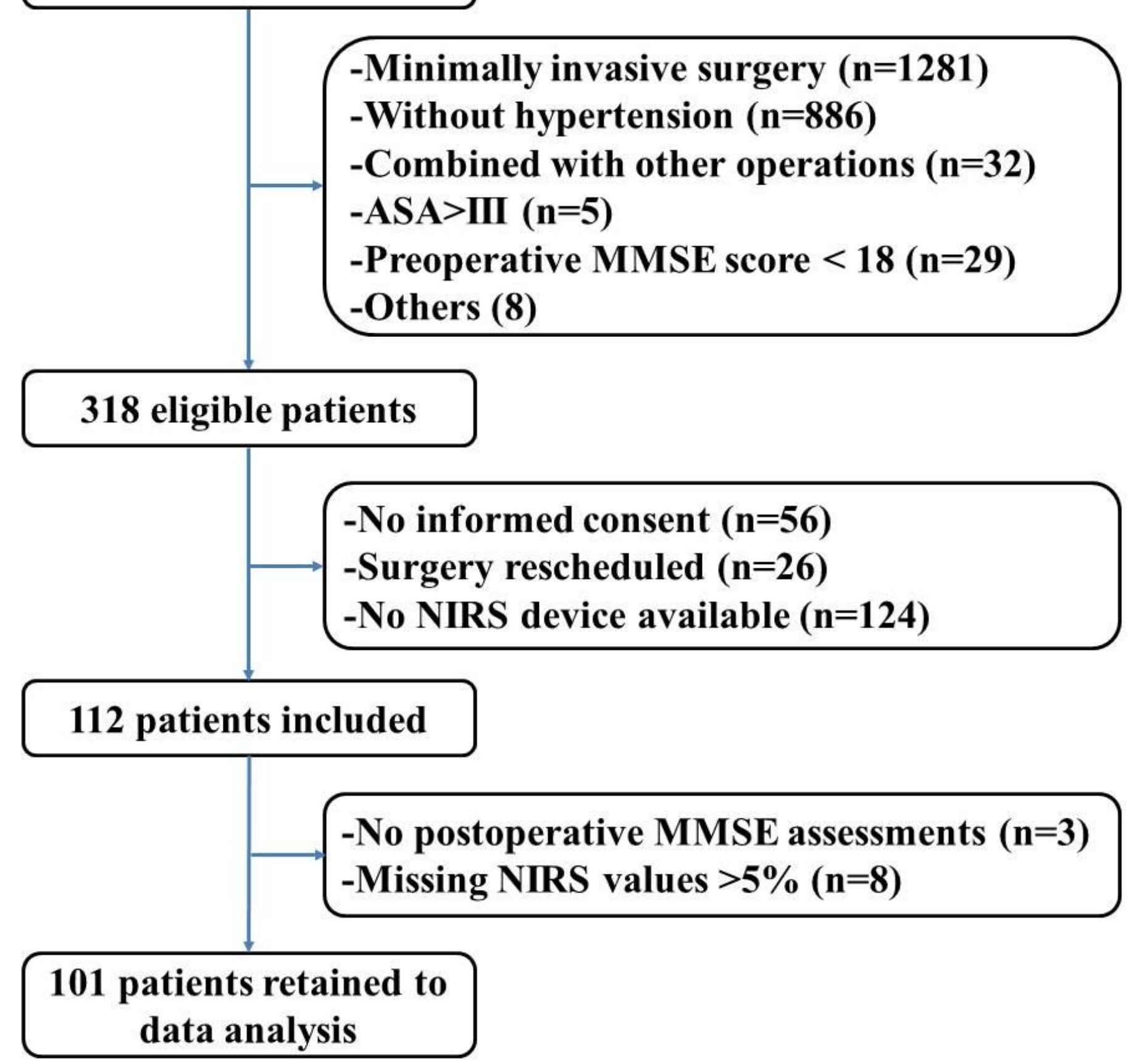

Figure 1

Flow diagram presenting patient enrolment; ASA, American Society of Anesthesiologists; MMSE, minimental state examination; NIRS, near-infrared spectroscopy 
a

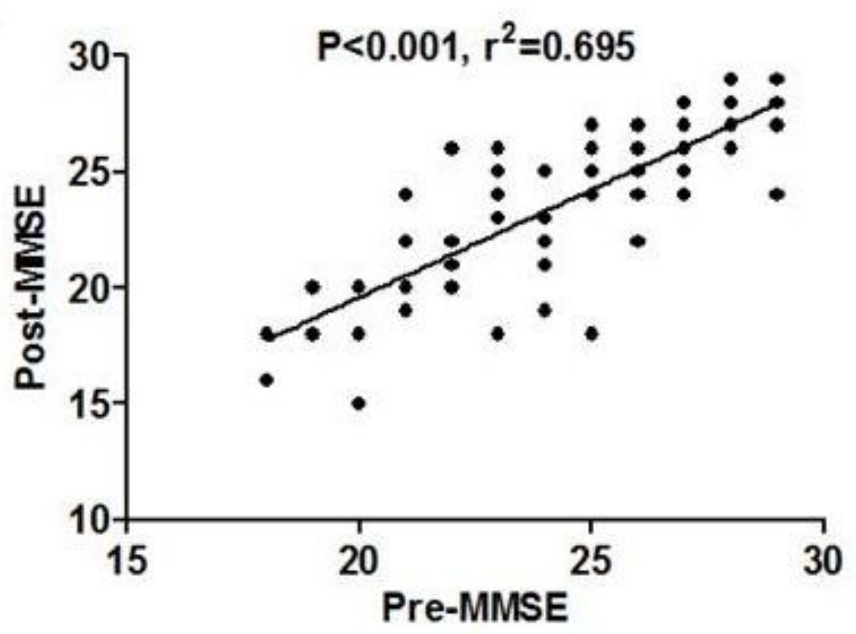

C

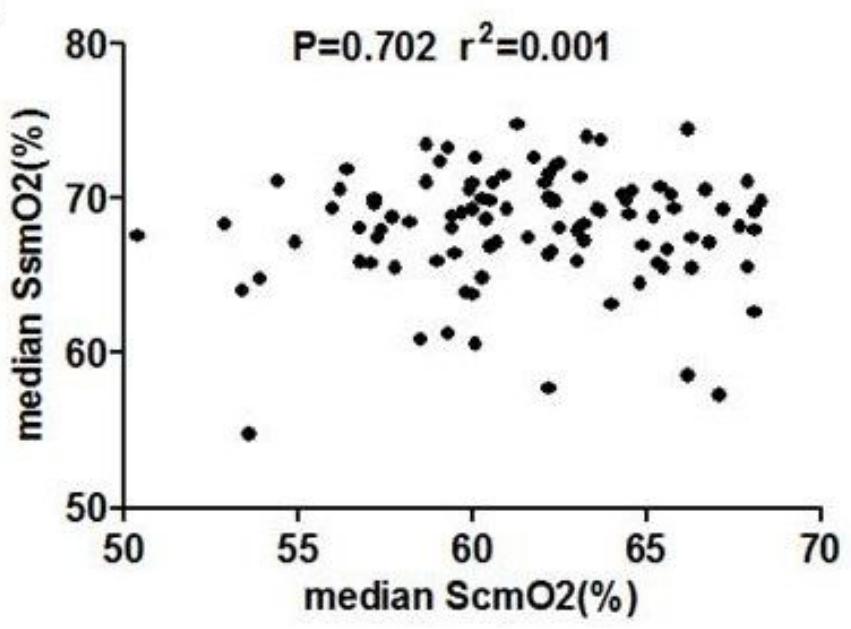

b

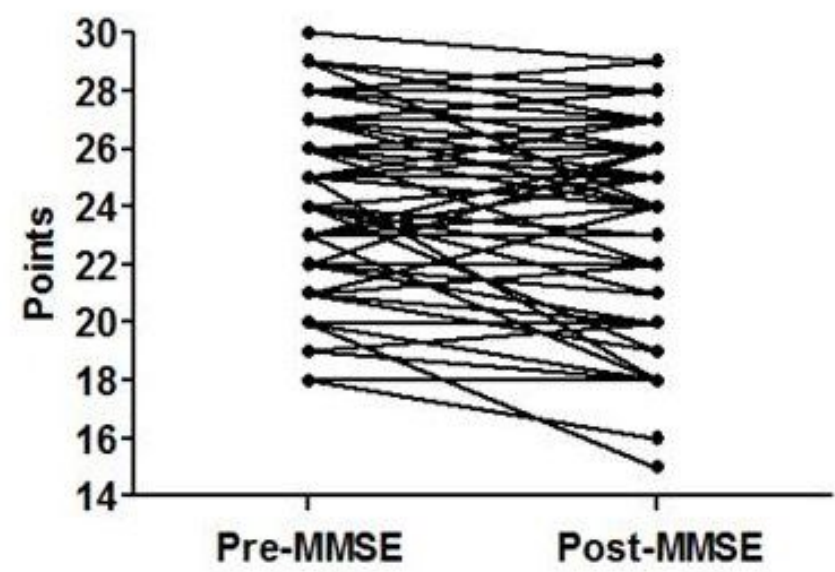

d

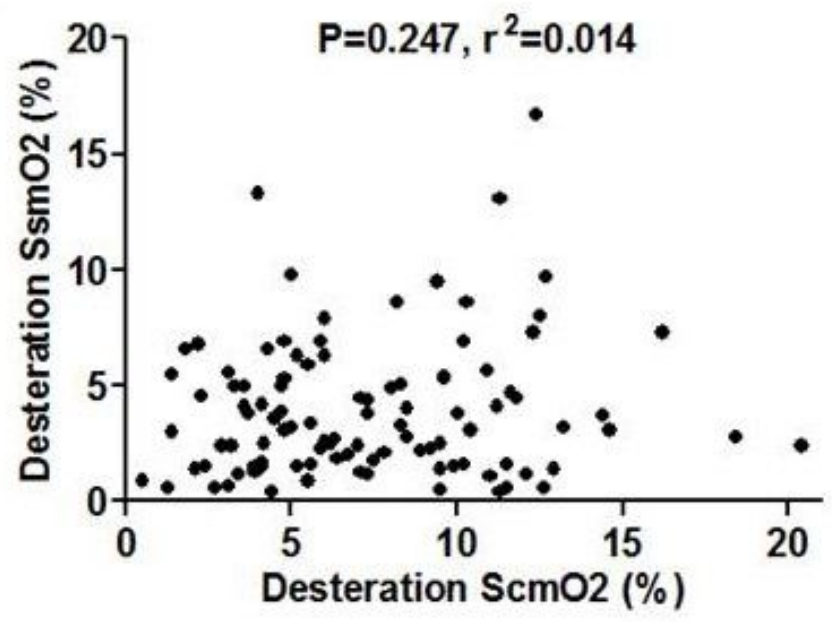

Figure 2

Characteristics of MMSE and tissue oxygen saturation parameters in 101 patients (a) The postoperative MMSE positively correlated with preoperative MMSE (b) Postoperative MMSE decrease significantly, compared with preoperative MMSE $(24.9 \pm 2.9$ vs. $24.1 \pm 3.3, \mathrm{P}<0.001)$ (c) No correlation between intraoperative cerebral oxygen saturation and somatic oxygen saturation (d) No correlation between intraoperative cerebral oxygen saturation declines and somatic oxygen saturation declines 
a

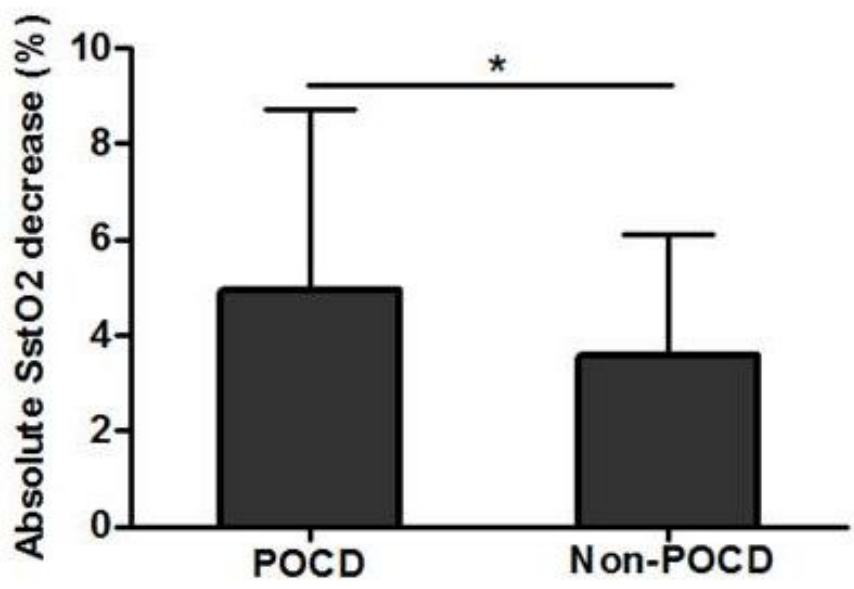

C

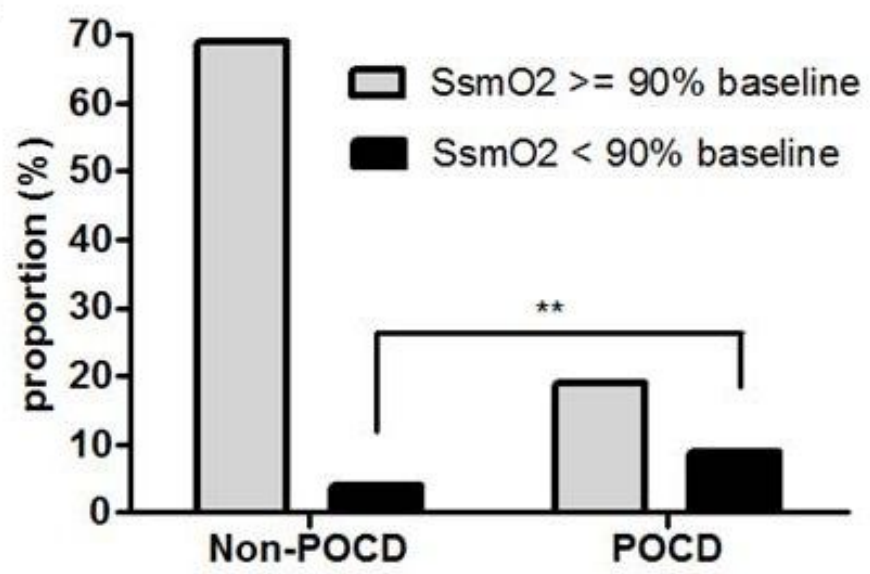

b

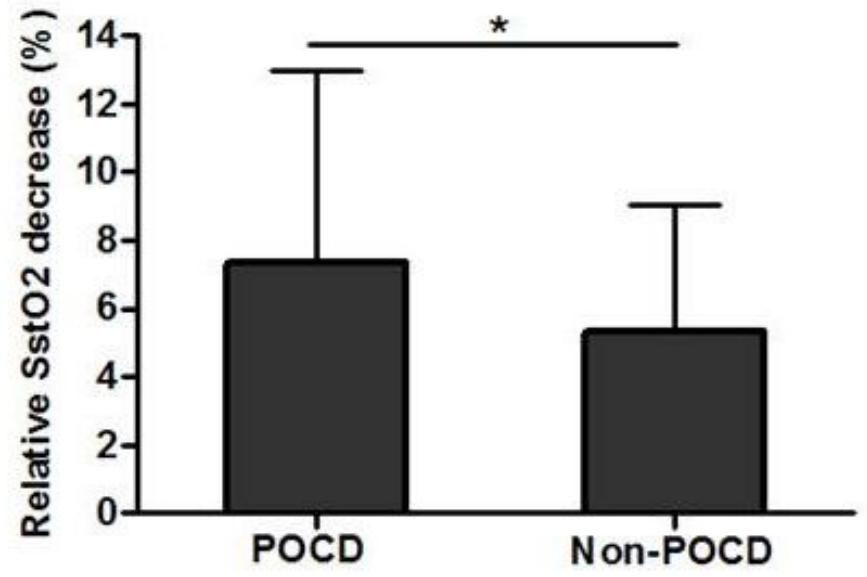

d

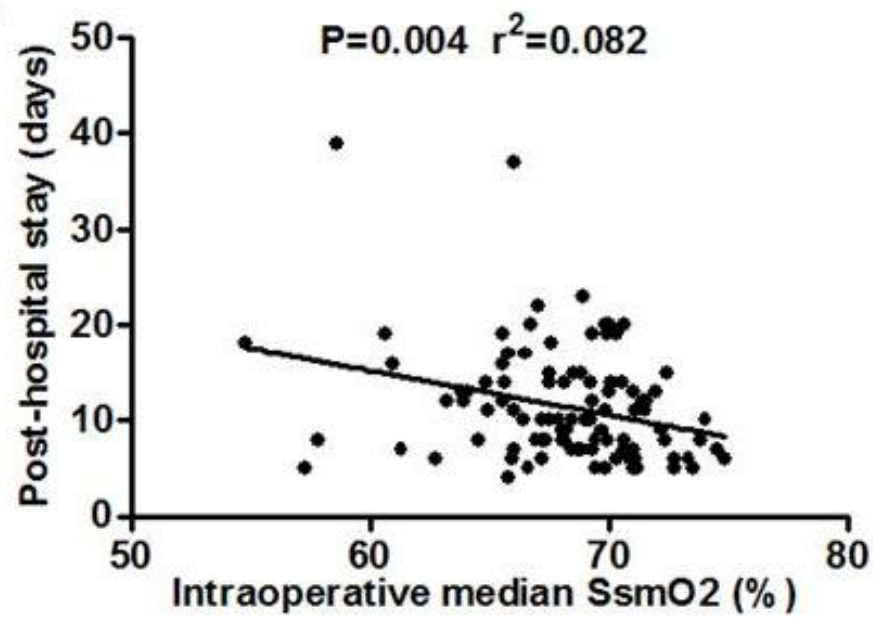

Figure 3

The association between Ssm02/Scm02 and POCD/postoperative hospital stay (a) The patients with POCD had a higher decrease in intraoperative absolute Sst02 decrease $(4.9 \pm 3.8$ vs. $3.6 \pm 2.6, P=0.037)(b)$ The patients with POCD had a higher decrease in intraoperative relative Sst02 decrease $(7.4 \pm 5.6$ vs. $5.3 \pm 3.8, P=0.036$ ) (c) The proportion below $90 \%$ of baseline Sst02 in the patients with POCD were significantly higher than patients without $(32.1 \%$ vs. $5.5 \%, \mathrm{P}<0.001)$, but most of the 28 patients with POCD did not experience intraoperative Sst02 desaturations below $90 \%$ of baseline (d) A negative correlation between the parameters of Sct02 (e.g., the absolute decrease) and the length of postoperative hospital stay $(\mathrm{r} 2=0.082, \mathrm{P}=0.004)$

\section{Supplementary Files}

This is a list of supplementary files associated with this preprint. Click to download.

- Supplementarytable.docx 